\title{
On the Zero-Set of Real Polynomials in Non-Separable Banach Spaces
}

\author{
By \\ Jesús FERRER*
}

\begin{abstract}
We show constructively that every homogeneous polynomial that is weakly continuous on the bounded subsets of a real Banach space whose dual is not weak ${ }^{*}$ separable admits a closed linear subspace whose dual is not weak ${ }^{*}$-separable either where the polynomial vanishes. We also prove that the same can be said for vectorvalued polynomials. Finally, we study the validity of this result for continuous 2homogeneous polynomials.
\end{abstract}

\section{§1. Introduction}

Since A. Plichko and A. Zagorodnyuk showed in [9] that, in a complex infinite-dimensional Banach space, every homogeneous continuous scalar-valued polynomial vanishes in a linear subspace of infinite dimension, there has been some interest concerning the zero-set of continuous homogeneous polynomials mainly in two directions, one in trying to measure the size (finite dimension) of the zero-set comparing with the dimension of the whole space, see [3] and [4], and another one when studying the validity of the result for infinite-dimensional real spaces, see [3], [1] and [5].

As it has become usual in the theory of infinite-dimensional complex Analysis, see [7], given a real Banach space $X$ and a positive integer $n$, by $\mathcal{P}\left({ }^{n} X\right)$ we

Communicated by H. Okamoto. Received February 20, 2006. Revised November 22, 2006.

2000 Mathematics Subject Classification(s): 47H60, 46B26.

Key words: Weakly continuous polynomials, zero-set, $\mathrm{w}^{*}$-separable dual.

The author has been partially supported by MEC and FEDER Project MTM2005-08210.

* Departamento de Análisis Matemático, Universidad de Valencia, Dr. Moliner, 50, 46100

Burjasot (Valencia), Spain.

e-mail: Jesus.Ferrer@uv.es

(c) 2007 Research Institute for Mathematical Sciences, Kyoto University. All rights reserved. 
shall represent the space of real-valued continuous homogeneous polynomials of degree $n$ endowed with its usual norm

$$
\|P\|=\sup \{|P(x)|:\|x\| \leq 1\} .
$$

For $P \in \mathcal{P}\left({ }^{n} X\right)$, there is by definition a continuous symmetric $n$-linear functional, usually denoted by $\stackrel{\vee}{P}$, such that $P(x)=\stackrel{\vee}{P}(x, x, \stackrel{(n)}{*}, x)$, the Polarization formula guarantees the uniqueness of such functional $\stackrel{\vee}{P}$. By $\mathcal{P}_{f}\left({ }^{n} X\right)$ we denote the subspace of $\mathcal{P}\left({ }^{n} X\right)$ formed by those polynomials which can be written as $P(x)=\sum_{j=1}^{m} \lambda_{j}\left\langle u_{j}^{*}, x\right\rangle^{n}$, with $\lambda_{j} \in \mathbb{R}, u_{j}^{*} \in X^{*}, 1 \leq j \leq m$, and they are called finite type polynomials. The space of approximable polynomials, $\mathcal{P}_{A}\left({ }^{n} X\right)$, is given by the closure of $\mathcal{P}_{f}\left({ }^{n} X\right)$ in $\mathcal{P}\left({ }^{n} X\right)$. By $\mathcal{P}_{w}\left({ }^{n} X\right)$ we represent the subspace of $\mathcal{P}\left({ }^{n} X\right)$ formed by those polynomials that are weakly continuous on the bounded subsets of $X$. A polynomial $P \in \mathcal{P}\left({ }^{n} X\right)$ is a nuclear polynomial whenever it has the form $P(x)=\sum_{j=1}^{\infty} a_{j}\left\langle u_{j}^{*}, x\right\rangle^{n}, x \in X$, where $\left(a_{j}\right)_{j=1}^{\infty} \in \ell_{1}$ and $\left(u_{j}^{*}\right)_{j=1}^{\infty}$ is a bounded sequence of $X^{*}$. Denoting by $\mathcal{P}_{N}\left({ }^{n} X\right)$ the class of nuclear polynomials, it is quite clear that

$$
\mathcal{P}_{f}\left({ }^{n} X\right) \subset \mathcal{P}_{N}\left({ }^{n} X\right) \subset \mathcal{P}_{A}\left({ }^{n} X\right) \subset \mathcal{P}_{w}\left({ }^{n} X\right) \subset \mathcal{P}\left({ }^{n} X\right) .
$$

In what follows $X$ will be an infinite-dimensional real Banach space and $X^{*}$ its topological dual. We use the symbol $\langle\cdot, \cdot\rangle$ to denote the standard duality between $X$ and $X^{*}$. If $P \in \mathcal{P}\left({ }^{n} X\right)$, its zero-set will be denoted by $P^{-1}(0)$, i.e., $P^{-1}(0)=\{x \in X: P(x)=0\}$. We say that $P^{-1}(0)$ has infinite dimension whenever it contains an infinite-dimensional linear subspace of $X$.

If $A \subset X$ and $B \subset X^{*}$, then we use the notation

$$
A^{\perp}=\left\{x^{*} \in X^{*}:\left\langle x^{*}, x\right\rangle=0, x \in A\right\}, \quad B_{\perp}=\left\{x \in X:\left\langle x^{*}, x\right\rangle=0, x^{*} \in B\right\} .
$$

For a polynomial $P \in \mathcal{P}\left({ }^{n} X\right)$, the following conjugacy relationship between its first and $(n-1)$-th derivatives turned out to be relevant. The first derivative is the mapping $P^{\prime}: X \longrightarrow X^{*}$ such that

$$
P^{\prime}(x)=n \stackrel{\vee}{P}\left(x,{ }^{(n-1)}, x, \cdot\right), \quad x \in X,
$$

while the $(n-1)$-th derivative is given by the continuous linear map $P^{(n-1)}$ : $X \longrightarrow \mathcal{L}_{s}\left(X^{n-1}\right)$ such that

$$
P^{(n-1)}(x)=n ! \stackrel{\vee}{P}\left(x, \cdot,{ }^{(n-\cdots)}, \cdot\right), \quad x \in X,
$$

where $\mathcal{L}_{s}\left(X^{n-1}\right)$ denotes the space of symmetric continuous $(n-1)$-linear functionals on $X$. It is then straightforward to notice, using the Polarization formula, that

$$
\operatorname{Ker} P^{(n-1)}=P^{\prime}(X)_{\perp}
$$


Notice that $\operatorname{Ker} P^{(n-1)}$ is a closed linear subspace of $P^{-1}(0)$ such that every maximal linear subspace of $P^{-1}(0)$ must contain it: If $Z$ is such a maximal subspace, then, for $x \in \operatorname{Ker} P^{(n-1)}, z \in Z$,

$$
\begin{aligned}
P(x+z)= & P(x)+P(z)+\sum_{j=1}^{n-1}\left(\begin{array}{c}
n \\
j
\end{array}\right) \stackrel{\vee}{P}\left(x, \stackrel{(j)}{P}, x, z,{ }^{(n-\cdots)}, z\right) \\
= & \frac{1}{n !} P^{(n-1)}(x)\left(x,{ }^{(n-1)}, x\right) \\
& +\sum_{j=1}^{n-1} \frac{1}{j !(n-j) !} P^{(n-1)}(x)\left(x,{ }^{(j-1)}, x, z,{ }^{(n-j)}, z\right)=0,
\end{aligned}
$$

i.e., $Z+\operatorname{Ker} P^{(n-1)} \subset P^{-1}(0)$, and the maximality of $Z$ yields that $\operatorname{Ker} P^{(n-1)}$ is contained in $Z$.

Hence, if $\operatorname{Ker} P^{(n-1)}$ were non-zero, we would easily obtain a non-zero linear subspace contained in $P^{-1}(0)$. Indeed, we will seek for conditions in order to guarantee that $\operatorname{Ker} P^{(n-1)}$ is sufficiently big. For this purpose, recall that

$$
\left(\operatorname{Ker} P^{(n-1)}\right)^{\perp}=\left(P^{\prime}(X)_{\perp}\right)^{\perp}=\overline{\operatorname{lin}}^{w^{*}}\left(P^{\prime}(X)\right),
$$

and so, roughly speaking, the smaller $P^{\prime}(X)$ is the bigger $\operatorname{Ker} P^{(n-1)}$ will be. In particular, if $P^{\prime}(X)$ were separable, then $\left(X / \operatorname{Ker} P^{(n-1)}\right)^{*}=\left(\operatorname{Ker} P^{(n-1)}\right)^{\perp}$ would have to be weak*-separable and this is mainly the reason why in the next section we shall be dealing with this type of space.

\section{$\S 2 . \quad$ Spaces which can be Injected into a Hilbert Space}

In this section we proceed to introduce a class of real Banach spaces, and a subclass, which will help us in our goal of finding conditions under which the zero-set of a polynomial will contain big enough linear subspaces.

We say that a real Banach space $X$ is in class $\mathcal{C}_{H}$ whenever there exists a one-to-one continuous linear map from $X$ into a Hilbert space. When $X \in \mathcal{C}_{H}$ we shall say that $X$ is injected into a Hilbert space. If $X$ is injected into a separable Hilbert space, then we shall write $X \in \mathcal{W}^{*}$. Clearly, $\mathcal{W}^{*} \subset \mathcal{C}_{H}$. The following properties of the spaces in these two classes are quite straightforward.

Proposition 1. The following conditions are equivalent for a space $X$ :

(i) $X \in \mathcal{W}^{*}$.

(ii) $X^{*}$ is weak $k^{*}$-separable.

(iii) $X^{*}$ has a countable total subset. 
Proposition 2. If $X$ is in class $\mathcal{C}_{H}$ (respectively, in $\mathcal{W}^{*}$ ) and $Y$ is a space that is injected linearly and continuously into $X$, then $Y \in \mathcal{C}_{H}$ (respectively, $\left.Y \in \mathcal{W}^{*}\right)$. Hence, every closed linear subspace of $X$ is in the same class that $X$.

Proposition 3. If $X$ is separable, then $X$ and $X^{*}$ are in $\mathcal{W}^{*}$.

The next result is used several times in this paper.

Proposition 4. Let $Y$ be a closed linear subspace of the Banach space $X$. If $Y$ is in $\mathcal{W}^{*}$ and $X / Y$ is in $\mathcal{C}_{H}$, then $X$ is in $\mathcal{C}_{H}$.

Proof. With no loss of generality, we may assume that we have two oneto-one bounded linear maps

$$
S_{1}: Y \longrightarrow \ell_{2}, \quad S_{2}: X / Y \longrightarrow \ell_{2}\left(\Gamma_{0}\right),
$$

with $\Gamma_{0}$ being a set that is disjoint from the set of positive integers $\mathbb{N}$. Now, for each $j \in \mathbb{N}$, if $e_{j}$ denotes the corresponding unit vector, we have that $S_{1}^{*} e_{j} \in Y^{*}$. Let $v_{j}^{*} \in X^{*}$ be the extension of $S_{1}^{*} e_{j}$ to $\mathrm{X}$ such that $\left\|v_{j}^{*}\right\|=\left\|S_{1}^{*} e_{j}\right\|$. Setting $\Gamma:=\mathbb{N} \cup \Gamma_{0}$, we define the mapping $T: X \rightarrow \ell_{2}(\Gamma)$ such that, for $x \in X$, $T x:=\left(\lambda_{\gamma}\right)_{\gamma \in \Gamma}$ where

$$
\lambda_{\gamma}:=\left\{\begin{array}{l}
2^{-\gamma}\left\langle v_{\gamma}^{*}, x\right\rangle, \quad \gamma \in \mathbb{N}, \\
\left\langle S_{2}(x+Y), e_{\gamma}\right\rangle, \quad \gamma \in \Gamma_{0} .
\end{array}\right.
$$

Then, $T$ is a well defined linear map such that it is bounded. To see that it is one-to-one, let $x \in X$ be such that $T x=0$, then, $0=\left\langle S_{2}(x+Y), e_{\gamma}\right\rangle, \gamma \in \Gamma_{0}$, implies that $S_{2}(x+Y)=0$, and so $x \in Y$; hence, from $0=2^{-j}\left\langle v_{j}^{*}, x\right\rangle, j \in \mathbb{N}$, it follows that $0=\left\langle S_{1}^{*} e_{j}, x\right\rangle=\left\langle e_{j}, S_{1} x\right\rangle, j \in \mathbb{N}$, therefore $S_{1} x=0$, and $x=0$.

If in the proof of the former proposition $\Gamma_{0}$ is taken to be countable, then the next result obtains.

Corollary 1. Let $Y$ be a closed linear subspace of $X$ such that $Y$ and $X / Y$ are both in $\mathcal{W}^{*}$, then $X$ is also in $\mathcal{W}^{*}$, i.e., being in $\mathcal{W}^{*}$ is a three-space property.

Concerning the connection of the two classes introduced before with the problem of finding big linear subspaces inside the zero-set of a polynomial, we must indicate that this relationship already appears in [1] and [5]. Also, the problem of knowing whether, analogously to Corollary 1 , being in $\mathcal{C}_{H}$ is 
a three-space property is stated in [5], as well as the following particular case (in some sense conjugate to the statement of Proposition 4): "If $Y \in \mathcal{C}_{H}$ and $X / Y \in \mathcal{W}^{*}$, does this imply that $X \in \mathcal{C}_{H}$ ?". Admitting first that this author has been unable to answer none of the preceding problems, the class of spaces satisfying this latter property will be considered in our last section when we deal with continuous 2 -homogeneous polynomials.

We already know that, if $X$ is separable then $X$ and $X^{*}$ are both in $\mathcal{W}^{*}$, let's take a look now at some other examples of spaces not belonging to $\mathcal{W}^{*}$, which will obviously be non-separable. After [11, p. 600], we know that every non-separable weakly compactly generated space, and hence every non-separable reflexive one and $c_{0}(\Gamma), \Gamma$ an uncountable set, has a non-weak*separable dual. This, plus the fact that $c_{0}(\Gamma)$ can be canonically injected into $\ell_{\infty}(\Gamma)$, yields that, for uncountable $\Gamma, c_{0}(\Gamma)$ and $\ell_{\infty}(\Gamma)$ are not in $\mathcal{W}^{*}$ and clearly $\ell_{2}(\Gamma) \in \mathcal{C}_{H} \backslash \mathcal{W}^{*}$. The easiest example of a space $X$ such that $X \in \mathcal{W}^{*}$ and $X^{*} \notin \mathcal{W}^{*}$ is given by $X=\ell_{\infty}$ : Being obvious that $\ell_{\infty} \in \mathcal{W}^{*}$, we show that $\ell_{\infty}^{*} \notin \mathcal{W}^{*}$ : It is shown in [10] that $\ell_{\infty}$ contains a closed subspace $F$ such that $\ell_{\infty} / F$ is isomorphic to a non-separable Hilbert space. Hence, $F^{\perp}=\left(\ell_{\infty} / F\right)^{*}$ is a subspace of $\ell_{\infty}^{*}$ which is also isomorphic to a non-separable Hilbert space. If $\ell_{\infty}^{*}$ were in class $\mathcal{W}^{*}$, then, from Proposition 2, there would be a non-separable Hilbert space in $\mathcal{W}^{*}$, which is clearly contradictory.

There are also examples satisfying the contrary, i.e., $X \notin \mathcal{W}^{*}$ and $X^{*} \in$ $\mathcal{W}^{*}$. In particular, there is one which plays a somewhat outstanding role and we shall take a look at it right now. Let $X=c_{0}([0,1])$. Then $X^{*}=\ell_{1}([0,1])$, and to show that $X^{*}$ is in $\mathcal{W}^{*}$, since the space of continuous functions $C[0,1]$, being separable, is a quotient of $\ell_{1}$, and therefore its topological dual $C[0,1]^{*}$ is isomorphic to a subspace of $\ell_{\infty}$, it suffices to see that $\ell_{1}([0,1])$ can be continuously injected into $C[0,1]^{*}$. This is done by noticing that the mapping $T: \ell_{1}([0,1]) \rightarrow C[0,1]^{*}$ such that, if $x=\left(x_{\gamma}\right) \in \ell_{1}([0,1]), T x:=\sum_{\gamma \in[0,1]} x_{\gamma} \delta_{\gamma}$, where $\delta_{\gamma}$ is the Dirac measure at the point $\gamma \in[0,1]$, is one-to-one bounded and linear.

Also, since $\left(\ell_{\infty} / c_{0}\right)^{*}$ admits no countable total subsets [8, p. 316], it follows that $\ell_{\infty} / c_{0}$ is not in $\mathcal{W}^{*}$.

We finish this section by showing that, if $X \notin \mathcal{W}^{*}$, then every sequence of closed linear subspaces $\left(E_{j}\right)_{j=1}^{\infty}$ such that $X / E_{j} \in \mathcal{W}^{*}, j \geq 1$, satisfies that $\cap_{j=1}^{\infty} E_{j} \notin \mathcal{W}^{*}$.

Lemma 1. Let $E$ be a closed linear subspace of the Banach space $X$. Then $E^{\perp}$ is $\sigma\left(X^{*}, X\right)$-separable if and only if there is a sequence $\left(u_{j}^{*}\right)_{j=1}^{\infty}$ in $X^{*}$ such that $E=\cap_{j=1}^{\infty} \operatorname{Ker} u_{j}^{*}$. 
Proposition 5. Let $\left(E_{j}\right)_{j=1}^{\infty}$ be a sequence of closed linear subspaces of $X$ such that, for each $j, E_{j}^{\perp}$ is $\sigma\left(X^{*}, X\right)$-separable. Let $E:=\cap_{j=1}^{\infty} E_{j}$, then:

(i) $\quad E^{\perp}$ is also $\sigma\left(X^{*}, X\right)$-separable.

(ii) If $X \notin \mathcal{W}^{*}$, then $E \notin \mathcal{W}^{*}$.

Proof. For each $j$, from the previous lemma, there is a sequence $\left(u_{j k}^{*}\right)_{k=1}^{\infty}$ $\subset X^{*}$ such that $E_{j}=\cap_{k=1}^{\infty} \operatorname{Ker} u_{j k}^{*}$. Hence

$$
E^{\perp}=\left(\cap_{j=1}^{\infty} E_{j}\right)^{\perp}=\left(\cap_{j, k=1}^{\infty} \operatorname{Ker} u_{j k}^{*}\right)^{\perp}=\overline{l i n}^{w^{*}}\left\{u_{j k}^{*}: j, k \geq 1\right\}
$$

is clearly $\sigma\left(X^{*}, X\right)$-separable, thus obtaining (i). Besides, this yields $X / E \in$ $\mathcal{W}^{*}$, and, if $X \notin \mathcal{W}^{*}$, the 3 -space property shown in Corollary 1 guarantees (ii).

\section{§3. Zero-Sets of Weakly Continuous Polynomials}

In this section we make use of the previous results to show that, for every homogeneous polynomial of an arbitrary degree that is weakly continuous on the bounded subsets of a space $X$ not belonging to class $\mathcal{W}^{*}$, its zero-set contains a closed linear subspace $Z$ which is not in $\mathcal{W}^{*}$ either, i.e., its dual is not weak*-separable, and so $Z$ is clearly non-separable. This provides a strict generalization of some results in [1] and also analogs of some other results in $[5]$

Proposition 6. If $X$ is a Banach space which is not in class $\mathcal{W}^{*}$, then, if $n$ is any positive integer, for each $P \in \mathcal{P}_{w}\left({ }^{n} X\right)$, Ker $P^{(n-1)}$ is not in $\mathcal{W}^{*}$.

Proof. If $P \in \mathcal{P}_{w}\left({ }^{n} X\right)$, making use of the conjugacy relation mentioned in the first section, we have

$$
\operatorname{Ker} P^{(n-1)}=P^{\prime}(X)_{\perp} .
$$

From [7, p. 88, Proposition 2.6], we know that $P^{\prime}$ is (weak-to-norm)-uniformly continuous on the bounded subsets and, since $B_{X}$ is weakly precompact, it follows that $P^{\prime}(X)$ is norm-separable in $X^{*}$. Clearly then, $\overline{\operatorname{lin}} w^{*}\left(P^{\prime}(X)\right)$ is weak*-separable and so, since

$$
\left(X / P^{\prime}(X)_{\perp}\right)^{*}=\left(P^{\prime}(X)_{\perp}\right)^{\perp}=\overline{\operatorname{lin}}^{w^{*}}\left(P^{\prime}(X)\right),
$$

we have that $X / P^{\prime}(X)_{\perp}$ is in $\mathcal{W}^{*}$. From Corollary 1 , since $X$ is not in $\mathcal{W}^{*}$, it follows that $\operatorname{Ker} P^{(n-1)}=P^{\prime}(X)_{\perp}$ is not in $\mathcal{W}^{*}$.

Recalling that $\operatorname{Ker} P^{(n-1)}$ is contained in every maximal linear subspace contained in $P^{-1}(0)$, the next result clearly follows. 
Corollary 2. If $X \notin \mathcal{W}^{*}$, then, for every integer $n$ and every $P \in$ $\mathcal{P}_{w}\left({ }^{n} X\right)$, every maximal linear subspace $Z$ contained in $P^{-1}(0)$ is such that $Z \notin \mathcal{W}^{*}$.

The next result gives us another characterization of the spaces in class $\mathcal{W}^{*}$.

Corollary 3. For a Banach space $X$, the following conditions are equivalent:

(i) $\quad X \in \mathcal{W}^{*}$.

(ii) For any even integer $n, X$ admits a positive definite polynomial $P \in \mathcal{P}_{N}\left({ }^{n} X\right)$.

(iii) For any even integer $n, X$ admits a positive definite polynomial $P \in \mathcal{P}_{w}\left({ }^{n} X\right)$.

(iv) There is an even integer $n$ such that $X$ admits a positive definite polynomial $P \in \mathcal{P}_{w}\left({ }^{n} X\right)$.

(v) There is an even integer $n$ such that $X$ admits a positive definite polynomial $P \in \mathcal{P}_{N}\left({ }^{n} X\right)$.

Proof. If $X \in \mathcal{W}^{*}$, let $T: X \longrightarrow \ell_{2}$ be a one-to-one continuous linear map. For any even $n$, the $n^{\text {th }}$-degree polynomial

$$
P(x):=\sum_{j=1}^{\infty} 2^{-j}\left\langle e_{j}, T x\right\rangle^{n}=\sum_{j=1}^{\infty} 2^{-j}\left\langle T^{*} e_{j}, x\right\rangle^{n}, \quad x \in X,
$$

is clearly nuclear and positive definite, hence we have that (i) $\Rightarrow$ (ii).

Since (ii) $\Rightarrow$ (iii) and (iii) $\Rightarrow$ (iv) are obvious, we see that (iv) $\Rightarrow$ (v): Under condition (iv), $X$ has to be in $\mathcal{W}^{*}$, otherwise, after Corollary 2, no polynomial of $\mathcal{P}_{w}\left({ }^{n} X\right)$ would be positive definite; hence, after (i) $\Rightarrow$ (ii), (v) follows.

(v) $\Rightarrow$ (i): Obvious, after Corollary 2 .

As a by-product of this last corollary, we obtain a stronger version of part (i) in Theorem 16 of [1].

Corollary 4. Let $X$ be any infinite-dimensional real Banach space. Then, either $X$ admits a positive definite nuclear polynomial of degree 2, or, for every positive integer $n$, the zero-set of every $P \in \mathcal{P}_{w}\left({ }^{n} X\right)$ contains a closed linear subspace of $X$ whose dual is not weak*-separable.

Proof. For any space $X$, if $X$ is class $\mathcal{W}^{*}$, from Corollary 3 , there is a positive definite nuclear polynomial of any even degree, in particular of the 
$2^{\text {nd }}$ degree. On the other hand, if $X$ is not in $\mathcal{W}^{*}$, from Proposition 6, for arbitrary $n$, if $P \in \mathcal{P}_{w}\left({ }^{n} X\right)$, then $\operatorname{Ker} P^{(n-1)}$ is not in class $\mathcal{W}^{*}$ either, and, since $\operatorname{Ker} P^{(n-1)} \subset P^{-1}(0)$, the result follows.

The results previously obtained will be used in the following to show that, if $X \notin \mathcal{W}^{*}$, then every vector-valued polynomial, not necessarily homogeneous, which is weakly continuous on the bounded subsets of $X$ admits a closed linear subspace not belonging to $\mathcal{W}^{*}$ where the polynomial is constant.

Lemma 2. If $P \in \mathcal{P}_{w}\left({ }^{n} X\right)$, then $\left(\operatorname{Ker} P^{(n-1)}\right)^{\perp}$ is $\sigma\left(X^{*}, X\right)$-separable.

Proof. Repeating the argument used in the beginning of the proof of Proposition 6 , we have that $P^{\prime}(X)$ is separable in $X^{*}$ and thus

$$
\left(\operatorname{Ker} P^{(n-1)}\right)^{\perp}=\left(P^{\prime}(X)_{\perp}\right)^{\perp}=\overline{\operatorname{lin}}^{w^{*}} P^{\prime}(X)
$$

is $\sigma\left(X^{*}, X\right)$-separable.

Proposition 7. Let $\left(n_{j}\right)_{j=1}^{\infty}$ be a sequence of positive integers and let $\left(P_{j}\right)_{j=1}^{\infty}$ be a sequence of polynomials such that, for each $j, P_{j} \in \mathcal{P}_{w}\left({ }^{n_{j}} X\right)$. If $X \notin \mathcal{W}^{*}$, then there is a closed linear subspace $Z$ in $X$ such that $Z \notin \mathcal{W}^{*}$ and $Z \subset \cap_{j=1}^{\infty} P_{j}^{-1}(0)$.

Proof. For each $j$, set $Z_{j}:=\operatorname{Ker} P_{j}^{\left(n_{j}-1\right)}$. Then, from the previous lemma, we have that $Z_{j}^{\perp}$ is $\sigma\left(X^{*}, X\right)$-separable, $j \geq 1$. Setting $Z:=\cap_{j=1}^{\infty} Z_{j}$, we know from Proposition 5 that $Z^{\perp}$ is $\sigma\left(X^{*}, X\right)$-separable, and so, since $X \notin \mathcal{W}^{*}$, we have that $Z \notin \mathcal{W}^{*}$. Now, since it is evident that $\operatorname{Ker} P_{j}^{\left(n_{j}-1\right)} \subset P_{j}^{-1}(0), j \geq 1$, the result follows.

For a Banach space $Y$ and a positive integer $n$, the symbols $\mathcal{P}\left({ }^{n} X, Y\right)$ and $\mathcal{P}_{w}\left({ }^{n} X, Y\right)$ will denote the spaces of $n$-homogeneous continuous polynomials on $X$ with values in $Y$ and the subspace formed by those which are weakly continuous (to say it in a more explicit way, weak-to-norm continuous) on the bounded subsets of $X$, respectively. We see next that, when $X$ is not in class $\mathcal{W}^{*}$, any countable family of polynomials in $\mathcal{P}_{w}\left({ }^{n} X, Y\right)$ vanishes simultaneously on quite a big linear subspace.

Corollary 5. Let $X, Y$ be Banach spaces with $X \notin \mathcal{W}^{*}$. Let $\left(n_{j}\right)_{j=1}^{\infty}$ be a sequence of positive integers and $\left(P_{j}\right)_{j=1}^{\infty}$ a sequence of polynomials such that, for each $j, P_{j} \in \mathcal{P}_{w}\left({ }^{n_{j}} X, Y\right)$. Then there is a closed linear subspace $Z$ in $X$ such that $Z \notin \mathcal{W}^{*}$ and $P_{j_{\mid Z}}=0, j \geq 1$. 
Proof. For each $j$, again from [7, p. 88, Proposition 2.6], we know that $P_{j}$ is weak-to-norm uniformly continuous on the bounded subsets of $X$. Hence, we have that its range $P_{j}(X)$ is separable in $Y$. Thus, there is a separable closed linear subspace $Y_{0}$ in $Y$ such that $P_{j}(X) \subset Y_{0}, j \geq 1$. The separability of $Y_{0}$ guarantees the existence of a sequence $\left(v_{k}^{*}\right)_{k=1}^{\infty}$ in $Y^{*}$ such that $\left(\cap_{k=1}^{\infty} \operatorname{Ker} v_{k}^{*}\right) \cap$ $Y_{0}=\{0\}$. For $j, k \geq 1$, defining $Q_{j k}(x):=\left\langle v_{k}^{*}, P_{j}(x)\right\rangle, x \in X$, we obtain a polynomial $Q_{j k} \in \mathcal{P}_{w}\left({ }^{n_{j}} X\right)$. For $j \geq 1$, setting $Z_{j}:=\cap_{k=1}^{\infty} \operatorname{Ker} Q_{j k}^{\left(n_{j}-1\right)}$, after the proof of the previous proposition, we have that $Z_{j}^{\perp}$ is $\sigma\left(X^{*}, X\right)$ separable. Hence, from Proposition 5 , it follows that $Z:=\cap_{j=1}^{\infty} Z_{j}$ is such that $Z^{\perp}$ is $\sigma\left(X^{*}, X\right)$-separable. Consequently, since $X \notin \mathcal{W}^{*}, Z$ is neither in $\mathcal{W}^{*}$ and, if $x \in Z$, for $j \geq 1,\left\langle v_{k}^{*}, P_{j}(x)\right\rangle=Q_{j k}(x)=0, k \geq 1$, implies that $P_{j}(x) \in\left(\cap_{k=1}^{\infty} \operatorname{Ker} v_{k}^{*}\right) \cap Y_{0}$, i.e., $P_{j}(x)=0$.

Corollary 6. Let $P: X \longrightarrow Y$ be a polynomial, not necessarily homogeneous, which is weakly continuous on the bounded subsets of $X$. If $X \notin \mathcal{W}^{*}$, then there is a closed linear subspace $Z$ in $X$ such that $Z \notin \mathcal{W}^{*}$ and $P_{\mid Z}=P(0)$.

Proof. Let $P(x)=P(0)+\sum_{j=1}^{n} P_{j}(x), x \in X$, where $P_{j} \in \mathcal{P}_{w}\left({ }^{j} X, Y\right)$, $1 \leq j \leq n$. Applying the former corollary to the finite sequence $P_{1}, P_{2}, \ldots, P_{n}$, there is a closed linear subspace $Z$ in $X$ such that $Z \notin \mathcal{W}^{*}$ and $P_{j_{\mid Z}}=0$, $1 \leq j \leq n$. This clearly yields $P(x)=P(0), x \in Z$.

In the results previously given we determine constructively the big linear subspace contained in the polynomial's zero-set. Nevertheless, noticing that what we really use is that weak zero-neighborhoods contain finite-codimensional linear subspaces, there is a natural extension of these existence results to a larger frame, namely that of the mappings which are weak-to-norm continuous on the bounded sets. More explicitelly, we have the following generalization.

Corollary 7. Let $f: X \longrightarrow Y$ be a weak-to-norm continuous mapping on the bounded subsets of $X$ such that $f(0)=0$. If $X \notin \mathcal{W}^{*}$, then there is a closed linear subspace $Z$ in $X$, with $Z \notin \mathcal{W}^{*}$, such that $Z \subset f^{-1}(0)$.

Proof. Let $B_{X}$ and $B_{Y}$ denote the closed unit balls of $X$ and $Y$, respectively. After the previous consideration, for every pair of positive integers $m, n$, there is a closed finite-codimensional linear subspace $Z_{m n} \subset X$ such that

$$
Z_{m n} \cap n B_{X} \subset f^{-1}\left(\frac{1}{m} B_{Y}\right) \cap n B_{X} .
$$

Clearly, after Proposition 5, the subspace $Z:=\cap_{m, n} Z_{m n}$ does the job. 
Corollary 8. Let $\left(f_{j}\right)_{j=1}^{\infty}$ be a sequence of mappings from $X$ into $Y$ which are weak-to-norm continuous on the bounded subsets of $X$ and such that $f_{j}(0)=0, j \geq 1$. Assume that, for all $x \in X, f(x):=\lim _{j} f_{j}(x)$ exists. If $X \notin \mathcal{W}^{*}$, then there is a closed linear subspace $Z \subset X$ such that $Z \notin \mathcal{W}^{*}$ and $Z \subset f^{-1}(0)$.

As a consequence of the above corollary, since the polynomials of the Taylor expansion of an analytic map which is also weak-to-norm continuous on the bounded subsets can be seen to be also weak-to-norm continuous on bounded sets, see [2, Lemma 2.1], the next result obtains.

Corollary 9. Let $f: X \longrightarrow Y$ be an analytic map which is weak-tonorm continuous on the bounded subsets of $X$ and such that $f(0)=0$. If $X \notin \mathcal{W}^{*}$, then there is a closed linear subspace $Z$ in $X$, with $Z \notin \mathcal{W}^{*}$, such that $Z \subset f^{-1}(0)$.

\section{$\S 4$. Zero-Sets of Continuous 2-Homogeneous Polynomials}

The following characterization of the spaces in $\mathcal{C}_{H}$ is given in [1]: " $X \in \mathcal{C}_{H}$ if and only if $X$ admits a positive definite continuous 2-homogeneous polynomial". It is then proved there that, if $X \notin \mathcal{C}_{H}$, then every polynomial $P \in \mathcal{P}\left({ }^{2} X\right)$ has an infinite-dimensional zero-set and the following conjecture is stated

Conjecture. For a real Banach space $X$, either $X \in \mathcal{C}_{H}$, or, for every $P \in \mathcal{P}\left({ }^{2} X\right), P^{-1}(0)$ contains a non-separable linear subspace.

Also in that same paper, the authors prove the conjecture to be true when $X$ is of type 2 , and also when $X$ admits no positive definite continuous 4homogeneous polynomial. In what follows, we give some sufficient conditions under which the conjecture also holds.

Proposition 8. Let $X$ be a space such that $X \notin \mathcal{C}_{H}$ and $X^{*} \in \mathcal{C}_{H}$. Then, if $P \in \mathcal{P}\left({ }^{2} X\right)$, Ker $P^{\prime} \notin \mathcal{W}^{*}$.

Proof. The first Fréchet derivative of $P$ is the continuous linear map $P^{\prime}: X \rightarrow X^{*}$ such that $\left\langle P^{\prime}(x), y\right\rangle=2 \cdot \stackrel{\vee}{P}(x, y), x, y \in X$. Assuming $\operatorname{Ker} P^{\prime}$ were in $\mathcal{W}^{*}$, then, from Proposition 4 , since $X \notin \mathcal{C}_{H}$, we would have that $X / \operatorname{Ker} P^{\prime} \notin \mathcal{C}_{H}$. But the map $T: X / \operatorname{Ker} P^{\prime} \rightarrow X^{*}$ given by $T\left(x+\operatorname{Ker} P^{\prime}\right):=$ $P^{\prime}(x)$ is well defined linear bounded and one-to-one, which would imply that $X / \operatorname{Ker} P^{\prime}$ is injected into $X^{*}$, but $X^{*} \in \mathcal{C}_{H}$, after Proposition 2, would then yield $X / \operatorname{Ker} P^{\prime} \in \mathcal{C}_{H}$, a contradiction. 
Corollary 10. If $X \notin \mathcal{C}_{H}$ and $X^{*} \in \mathcal{C}_{H}$, then, for every $P \in \mathcal{P}\left({ }^{2} X\right)$, every maximal linear subspace $Z$ contained in $P^{-1}(0)$ is such that $Z \notin \mathcal{W}^{*}$.

We show next that, for uncountable $\Gamma$, the spaces $c_{0}(\Gamma), \ell_{p}(\Gamma), 2<p<\infty$, are of the type just considered, i.e., $X \notin \mathcal{C}_{H}, X^{*} \in \mathcal{C}_{H}$.

Lemma 3. Let $\Gamma$ be an uncountable set. Then, for $1 \leq p \leq 2$, the space $\ell_{p}(\Gamma) \in \mathcal{C}_{H}$, while, for $2<p \leq \infty, \ell_{p}(\Gamma) \notin \mathcal{C}_{H}$.

Proof. For $1 \leq p \leq 2$, it can be easily seen that the identity map from $\ell_{p}(\Gamma)$ into $\ell_{2}(\Gamma)$ is well defined linear bounded and one-to-one, thus $\ell_{p}(\Gamma) \in \mathcal{C}_{H}$. Now, to see that, for $2<p<\infty, \ell_{p}(\Gamma) \notin \mathcal{C}_{H}$, let $T: \ell_{p}(\Gamma) \rightarrow \ell_{2}(\Delta)$ be a bounded linear map, where $\Delta$ is any set. For each $\varepsilon>0$, the set $\Gamma_{\varepsilon}:=\{\gamma \in$ $\left.\Gamma:\left\|T e_{\gamma}\right\|>\varepsilon\right\}$ is finite, otherwise, we could find a sequence of distinct terms $\left(\gamma_{j}\right)_{j=1}^{\infty} \subset \Gamma_{\varepsilon}$. Let $E$ be the closed linear span of $\left(e_{\gamma_{j}}\right)_{j=1}^{\infty}$ in $\ell_{p}(\Gamma)$, thus we have that $E$ is a copy of $\ell_{p}$ inside $\ell_{p}(\Gamma)$. Now, after Pitt's theorem, the map $T_{\mid E}$ is compact and, since the sequence $\left(e_{\gamma_{j}}\right)_{j=1}^{\infty}$ is weakly null, the sequence $\left(T e_{\gamma_{j}}\right)_{j=1}^{\infty}$ converges to zero, which is clearly a contradiction. Hence, the set $\left\{\gamma \in \Gamma: T e_{\gamma} \neq 0\right\}$ is countable and, since $\Gamma$ is not countable, there is $\gamma \in \Gamma$ such that $T e_{\gamma}=0$, i.e., $e_{\gamma} \in \operatorname{Ker} T$ and so $T$ cannot be one-to-one. The same argument shows that $c_{0}(\Gamma) \notin \mathcal{C}_{H}$ and so $\ell_{\infty}(\Gamma) \notin \mathcal{C}_{H}$.

Corollary 11. Let $\Gamma$ be an uncountable set and let $X$ be any of the spaces $\ell_{p}(\Gamma), 2<p<\infty$, or $c_{0}(\Gamma)$. If $P$ is a continuous 2-homogeneous polynomial on $X$, then $\operatorname{Ker} P^{\prime}$ is a closed linear subspace contained in $P^{-1}(0)$ whose dual is not weak*-separable. Consequently, every maximal linear subspace contained in $P^{-1}(0)$ has a dual which is not weak ${ }^{*}$-separable. If $X=\ell_{\infty}(\Gamma)$, then, for every $P \in \mathcal{P}\left({ }^{2} X\right), P^{-1}(0)$ contains a closed linear subspace $Z$ such that $Z \notin \mathcal{W}^{*}$.

In [5, Proposition 6], it is shown that the above stated conjecture holds for spaces with the Controlled Separable Projection Property (CSPP), a class that contains the weakly compactly generated spaces and thus it contains the spaces $c_{0}(\Gamma), l_{p}(\Gamma), 2<p<\infty$. Therefore, the statement in Corollary 11 follows from the result before referred. Nevertheless, we would like to point out that, although this author does not know of any example of a space $X$ such that $X \notin \mathcal{C}_{H}, X^{*} \in \mathcal{C}_{H}$ and $X$ does not have the CSPP, still the result given in the previous corollary provides with a closed linear subspace, Ker $P^{\prime}$, contained in $P^{-1}(0)$ which is more than just non-separable, since it satisfies Ker $P^{\prime} \notin \mathcal{W}^{*}$.

To finish, we introduce another class of spaces. We say that a space $X$ is in class $\mathcal{C}_{H}^{\prime}$ whenever, for any sequence $\left(u_{j}^{*}\right)_{j=1}^{\infty}$ in $X^{*}$, we have that $\cap_{j=1}^{\infty} \operatorname{Ker} u_{j}^{*} \notin$ 
$\mathcal{C}_{H}$. Clearly, $\mathcal{C}_{H}$ and $\mathcal{C}_{H}^{\prime}$ are disjoint classes and we show that for the elements of class $\mathcal{C}_{H}^{\prime}$ the conjecture holds.

Proposition 9. Let $X \in \mathcal{C}_{H}^{\prime}$. If $P \in \mathcal{P}\left({ }^{2} X\right)$, then every maximal linear subspace contained in $P^{-1}(0)$ is non-separable.

Proof. Let $Z$ be one of such maximal subspaces and suppose it is separable. Let $Y:=P^{\prime}(Z)_{\perp}$. Then, by the maximality of $Z$, we have that $P^{-1}(0) \cap Y=Z$ and $P$ does not change sign in $Y$ (we shall assume that $\left.P_{\mid Y} \geq 0\right)$.

Since $Y^{\perp}={\overline{P^{\prime}(Z)}}^{w^{*}}$ is $\sigma\left(X^{*}, X\right)$-separable, after Lemma 1 we have that there is a sequence $\left(u_{j}^{*}\right)_{j=1}^{\infty}$ in $X^{*}$ such that $Y=\cap_{j=1}^{\infty} \operatorname{Ker} u_{j}^{*}$. Thus, since $X \in \mathcal{C}_{H}^{\prime}$, it follows that $Y \notin \mathcal{C}_{H}$. Now, by defining

$$
Q(x+Z):=P(x), \quad x \in Y,
$$

we obtain a polynomial $Q \in \mathcal{P}\left({ }^{2}(Y / Z)\right)$ which is positive definite. This implies that $Y / Z \in \mathcal{C}_{H}$, but, $Z$ being separable yields $Z \in \mathcal{W}^{*}$, and so, after Proposition 4, we have that $Y \in \mathcal{C}_{H}$, a contradiction.

Finally, let us just remark that in order to solve positively the conjecture stated at the beginning of this last section, it would be sufficient to give a positive answer to the following question, which is equivalent to the second of the questions posed in [5, Remark 3]: If $X \notin \mathcal{C}_{H}$, and $\left(u_{j}^{*}\right)_{j=1}^{\infty} \subset X^{*}$, does it always follow that $\cap_{j=1}^{\infty} \operatorname{Ker} u_{j}^{*} \notin \mathcal{C}_{H}$ ?, i.e., is it true that $\neg \mathcal{C}_{H}=\mathcal{C}_{H}^{\prime}$ ?

\section{Acknowledgements}

This author would like to express his gratitude towards Professor M. Valdivia for his kindness and help when teaching him about the many weird properties that $\ell_{\infty}$ hides. Also it is a pleasure to thank Professor Vicente Montesinos for providing with a nice and simple proof of $c_{0}(\Gamma) \notin \mathcal{C}_{H}$. The author is also indebted with the referee for his valuable comments and simplifying arguments.

\section{References}

[1] R. M. Aron, C. Boyd, R. A. Ryan and I. Zalduendo, Zeros of polynomials on Banach spaces: the real story, Positivity 7 (2003), no. 4, 285-295.

[2] R. M. Aron, B. J. Cole and T. W. Gamelin, Weak-star continuous analytic functions, Canad. J. Math. 47 (1995), no. 4, 673-683. 
[3] R. Aron, R. Gonzalo and A. Zagorodnyuk, Zeros of real polynomials, Linear and Multilinear Algebra 48 (2000), no. 2, 107-115.

[4] R. M. Aron and M. P. Rueda, A problem concerning zero-subspaces of homogeneous polynomials, Linear Topol. Spaces Complex Anal. 3 (1997), 20-23.

[5] T. Banakh, A. Plichko and A. Zagorodnyuk, Zeros of quadratic functionals on nonseparable spaces, Colloq. Math. 100 (2004), no. 1, 141-147.

[6] J. Diestel, Sequences and series in Banach spaces, Springer, New York, 1984.

[7] S. Dineen, Complex analysis on infinite-dimensional spaces, Springer, London, 1999.

[8] G. J. O. Jameson, Topology and normed spaces, Chapman and Hall, London, 1974.

[9] A. Plichko and A. Zagorodnyuk, On automatic continuity and three problems of The Scottish book concerning the boundedness of polynomial functionals, J. Math. Anal. Appl. 220 (1998), no. 2, 477-494.

[10] H. P. Rosenthal, On quasi-complemented subspaces of Banach spaces, with an appendix on compactness of operators from $L^{p}(\mu)$ to $L^{r}(\nu)$, J. Functional Analysis 4 (1969), 176-214.

[11] I. Singer, Bases in Banach spaces. II, Editura Academiei Republicii Socialiste România, Bucharest, 1981. 